\title{
Measuring the Risk-Taking Willingness of Leaders and Non-Leaders Based on Prospect Theory: Differences in Decision-Making Between Two Groups
}

\author{
Jingchun Liang ${ }^{1 *}$
}

\author{
${ }^{I}$ Sun Yat-sen University \\ *Corresponding author. Email: 2469869717@qq.com
}

\begin{abstract}
Based on the Prospect Theory proposed by Daniel Kahneman and Amos Tversky (1979) on people's willingness in risk-taking, this article tries to find out the differences in the risk-taking willingness between two comparative groups, leaders and non-leaders, which are general in numerous fields. Therefore, an online survey was conducted through a questionnaire, where several simple questions related were designed to distinguish the subjects' attribute of leaders and non-leaders as well as their respective willingness in risk-taking. The result shows that when it comes to decision-making, the overall trend of people's attitudes towards risks is consistent with the description of the Prospect Theory, but leaders are more willing to take risks than non-leaders comparatively, both in gain versions and loss versions.
\end{abstract}

Keywords: Prospect Theory, Leaders and non-leaders, Risk-taking willingness, Decision-making.

\section{INTRODUCTION}

In 1944, Von Neumann and Morgenstern, based on the axiomatic hypothesis, put forward the Expected Utility Theory (EUT) with logical and mathematical tools, which is used to describe how a rational person makes an optimal decision under uncertain conditions. In 1979, Daniel Kahneman and Amos Tversky questioned the theoretical system of EUT and believed that individual decision-making is actually a choice of expectations and the result of the joint actions of various risks, in which special psychological processes and laws are often followed rather than various axioms assumed by EUT [1]. In short, Daniel Kahneman and Amos Tversky believed that people are often "irrational" when facing decisions, especially when facing loss. On this basis, they put forward the Prospect Theory (PT) in 1979 [2], in which one of the basic points is that people are risk-averse when facing gains and willing to take risks when facing losses (which is opposite to EUT). PT is one of the most important achievements in behavioural economics, and Kahneman therefore won the Nobel Prize in Economics in 2002[3]. PT has successfully predicted the phenomena resulting from specific decision behaviours in many different situations, such as corporate bond returns in the U.S. market [4], and an increasing number of algorithms based on PT have been developed for use in areas such as business analysis.

Leaders and non-leaders are two universal comparative groups in human society. In any organization or team, there is more or less a structure, either clear or vague, in which some people play the role of leaders and others play the role of non-leaders (or say, followers). There are some differences in personality and behaviour between leaders and non-leaders. A typical leader is usually considered to have an extroverted, open personality, a sense of responsibility [6], and always be ready to meet challenges. This being the case, it is hypothesized that leaders should be more willing to take risks when making decisions than non-leaders. This article attempts to qualitatively and simply judge a person's risk-taking willingness from the perspective of PT, and make a reasonable assumption in combination with the specific context of leader/non-leader: both leaders and non-leaders are risk-averse in GAIN and risk-taking in LOSS, but leaders are more inclined to take risks than non-leaders in both scenarios.

For a team, a decision is generally made collectively by the joint efforts of leaders and non-leaders, and in most cases, the opinions of leaders dominate. Therefore, 
understanding the decision-making behaviours and tendencies of team members, especially leaders, plays an important role in adjusting the decision-making model and making optimal decisions. For individuals, knowing about their preferences towards risks can also help them effectively avoid many decision traps and make better choices, no matter they are, or prefer to be leaders or non-leaders. Based on PT, this article may be able to provide a new direction for further researches on the behavioural differences between leaders and non-leaders through a simple survey about individual risk-taking willingness.

\section{CONDUCT A SURVEY}

In order to verify the correctness of the above hypothesis, I designed an online questionnaire for investigation. This survey is anonymous and filled out randomly to ensure privacy and fairness. The respondents are mainly my relatives, friends, classmates and their own friends, who have different professional and educational backgrounds. The survey was conducted from 21:40 February 16th, 2020 to 11:00 February 17th, 2020. A total of 102 valid questionnaires were collected with sufficient samples and strong validity, which could be used as original data for analysis.

\section{QUESTION DESIGN AND THE RESULTS}

The questionnaire consists of five multiple-choice questions, all of which are compulsory. There is no right or wrong answer. The questions are as follows:

Question 1. What is your gender?

A: Male B: Female

Question 2. In most cases, you prefer to be a?

A: leader B: non-leader

Question 3. According to the result of the previous question, to what extent would you prefer to be a leader/non-leader?

\section{A: High B: Medium C: Low}

Question 4. If you can make a profit from your stock, which of the following two situations is more acceptable for you?

\section{A: Get $\$ 1,000$ for sure \\ B: $50 \%$ probability to get $\$ 2,000,50 \%$ probability to get $\$ 0$}

Question 5. If the insurance company you run needs to pay compensation to customers, which of the following two situations is more acceptable for you?

A: Lose $\$ 1,000$ for sure
B: $50 \%$ probability to lose $\$ 2,000,50 \%$ probability to lose $\$ 0$

The following is a brief explanation of the reasons for setting these questions, and relative experimental results are presented. Question 1 determines the gender of the respondents and classifies them, and tries to reduce the impact of gender difference by controlling the number of male and female respondents to be close to each other. Question 2 identifies the leader/non-leader attribute of respondents to pave the way for the exploration of the difference in risk-taking willingness between leaders and non-leaders. It should be noted that "in most cases, you prefer to be..." is used in this question setting instead of directly asking respondents whether they are (ever) leaders/non-leaders. This is to avoid that: 1 . The respondents are unable to accurately identify their roles due to their multiple "identities" in different teams or different environments; 2. The respondents may not have an opportunity to become a leader yet, so they choose the option of not being a leader. But since they actually desire to be leaders and behave like leaders, it is obvious that these "potential" leaders should be included in the same category. There is additional evidence to back up the validity. An article survey shows that respondents who think they share characteristics with leaders in general feel more able to lead and seem more motivated to take over leadership positions as a result, confirming the role of leadership self-efficacy as a proximal antecedent of motivation to lead [5]. In other words, if one says "I'm motivated to be a leader," then he or she is more likely to be a leader, that is, to choose the "leader" option. And according to Badura [6], MTA can be divided into 3 types, but all of which can actually be linked to leadership effectiveness, either more or less directly. The responses to these two questions are as follows:

Table 1. Data of respondents' gender and leader/non-leader attribute

\begin{tabular}{cccc}
\hline Gender & Leaders & Non-leaders & Total \\
\hline male & 29 & 21 & 50 \\
\hline female & 23 & 29 & 52 \\
\hline total & 52 & 50 & 102 \\
\hline
\end{tabular}

As can be seen from Table 1, the gender ratio of respondents is balanced, and the number of people who are willing to be leaders and non-leaders is close to each other, which is an ideal result. Men are more likely to be leaders than women, but this is not the point that the article concerns so I'm not going into this issue.

Question 3 is closely related to Question 2, and its significance is to qualitatively reflect the respondents' tendency to become leaders/non-leaders. If one chooses medium or high, it is basically confirmed that he or she has a moderate will at least to be a leader/non-leader. If the choice is low, it is supposed that the boundary between leader and non-leader attribute is blurred for 
this respondent, which is not conducive to distinguishing. Therefore, if a large number of people choose "low", the result may be inaccurate. The responses to this question are as follows:

Table 2. Extent of willingness to become a leader/non-leader

\begin{tabular}{cccc}
\hline Extent & Men & Women & Total \\
\hline High & 10 & 13 & 31 \\
\hline Medium & 31 & 34 & 65 \\
\hline Low & 1 & 5 & 6 \\
\hline
\end{tabular}

Table 3. Respondents' choices in two conditions

\begin{tabular}{ccc}
\hline Condition & The safety option & The risk option \\
\hline Gain version & 73 & 29 \\
\hline Loss version & 29 & 73
\end{tabular}

As can be seen from Table 3, the overall trend well conform to PT, that is, most people, whether leaders or non-leaders, whether men or women, are risk-averse when faced with gains, but willing to take risks when faced with losses.
As can be seen from Table 2, only 6 respondents chose "low" (all from non-leaders), that is, most respondents have a certain tendency (medium or high) to be leaders or non-leaders. Therefore, the result obtained from Question 2 is basically accurate and valid.

The options of Questions 4 and 5 are based on the cases in the article about PT proposed by Daniel Kahneman and Amos Tversky in 1979 to qualitatively measure the risk-taking willingness of the respondents. The background of the questions are designed to make them more concrete by adding relevant conditions, which are not real. The responses to these two questions are as follows:

\section{RESULTS}

Based on the above results and data classification, the risk-taking willingness of leaders and non-leaders can be compared as follows:

Table 4. Risk-taking willingness of leaders and non-leaders

\begin{tabular}{ccccccc}
\hline Attribute & $\begin{array}{c}\text { The safety } \\
\text { option in } \\
\text { gain } \\
\text { version }\end{array}$ & $\begin{array}{c}\text { The risk } \\
\text { option in } \\
\text { gain } \\
\text { version }\end{array}$ & $\begin{array}{c}\text { Risk-taking } \\
\text { probability in } \\
\text { gain version }\end{array}$ & $\begin{array}{c}\text { The safety } \\
\text { option in } \\
\text { loss version }\end{array}$ & $\begin{array}{c}\text { The risk } \\
\text { option in } \\
\text { loss version }\end{array}$ & $\begin{array}{c}\text { Risk-taking } \\
\text { probability in loss } \\
\text { version }\end{array}$ \\
\hline Leaders & 33 & 19 & 0.37 & 13 & 39 & 0.75 \\
Non-leaders & 40 & 10 & 0.20 & 16 & 34 & 0.68
\end{tabular}

It can be seen from Table 4 that leaders have a higher preference for risk options than non-leaders in both gain version and loss version. In GAIN VERSION, the probability of leaders choosing to take risks is nearly 2 times that of non-leaders. In LOSE VERSION, the probability of leaders choosing to take risks is slightly higher than that of non-leaders by about $10 \%$.

The result of the survey on the risk-taking willingness of leaders and non-leaders confirms my hypothesis.

\section{DISCUSSION}

Jiang and his colleagues' findings[7] provide an angle to analyse why leaders tend to be more risk-taking than non-leaders. In this article, Jiang et al. divide respondents (leaders) into four types according to their desires to become leaders and their purposes to become leaders. They are explorers, climbers, conformists and visionaries (in one of his studies, there are only two of the overall 50 respondents who consider that they belong to none of these four groups, which affects the result little). It is shown in the figure below:

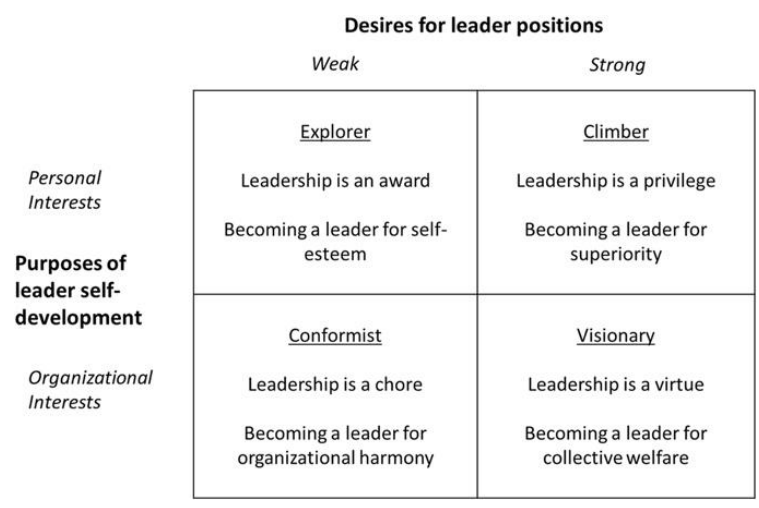

Figure 1. A leader self-developmental model of business leaders 
We should pay more attention to the leader groups with strong desires, because these groups correspond to "typical leaders" we have been concerned about above. Climbers, according to Jiang, are interested in attaining power and prestige from leadership positions. They see leadership as an important and prominent position within a group. They are passionate in becoming something of a celebrity which can gain prestige and raise their social status and naturally be well remunerated. They can adapt quickly to situational changes at work, and they are really keen on interpersonal relationships where they have to keep adjusting their strategies in getting along with different people and then trying to amuse them, which is rather tricky. It indicates that once an (underlying) crisis emerges, either in some crucial investments or relations with some important people, they are able to react quickly and make timely adjustments, so they are not that afraid of risks. Say, they may get $\$ 2,000$ rather than $\$ 1,000$ (as shown above) or lose nothing rather than $\$ 1,000$ (as shown above). Once the gamble wins, his or her decision-making ability will be affirmed, which will contribute to his or her career growth. For visionaries, life itself is an adventure full of fun and passion, so they never hesitate to break the status quo or to take risks in gaining an edge over the competition. That is, similar to climbers, they will not let go any chance to make their returns increase, even if it is just a tiny change. The different thing is that visionaries may focus more on collective rather than individual interests.

Explorers are less interested in gaining authority or control over others, but they think it important to stay in leadership positions as a way to affirm their worth and credentials. As a result, they are also trying to gain more by taking modest risks, although they are more nervous about whether a decision can cause harm to their career growth and they would be more vulnerable once something bad happens. As for conformists, they often choose to become leaders simply because their organizations or their bosses have open management positions that need filling. For most of them, there is no such thing as motivations. Hence, it is reasonable to exclude conformists from the group of typical leaders.

From analysis above, it can be found that apart from conformists, typical leaders (other three types of leaders) are more likely to take risks than non-leaders (as has been pointed out above, typical leaders are basically those who have a certain will to become leaders, that is, those who chose "medium" or "high" in Question 3 ). And since in most cases they take risks in order to increase returns, they are more motivated to take risks in gain versions than in loss versions.

\section{LIMITATIONS AND EXPECTATIONS}

Here I will discuss some possibly controversial aspects of the survey. First of all, although Questions 2 and 3 have enabled most people to identify themselves as leaders/non-leaders, which has been discussed in detail above, they are still not very rigorous. There may be some specific tests for measuring leader/non-leader attribute, such as survey conducted by Mcdaniel et al[8] in 2007 , but it would be rather cumbersome to add it in and respondents are prone to lose patience without enough payment (there is little remuneration for respondents). So it is not necessary to be so nit-picking. Similarly, Question 4 and Question 5 cannot fully represent the respondents' willingness to take risks. In fact, in Daniel Kahneman and Amos Tversky's article, there are many other conditions where the expected value is different between gain versions and loss versions (the expected value is lower in gain versions). The forms, amounts and corresponding probabilities of rewards or punishments are different to comprehensively measure the risk-taking willingness of the respondents. Questions 4 and 5 are only one specific pair of counterparts where the expected value is identical in both gain version and loss version. And for the same reason, only this model was selected to be a representative in the questionnaire to improve efficiency. Secondly, questionnaires were only collected from my friends, classmates, relatives and their own friends, and the interpersonal circle is still limited so the randomness is not strong enough. As mentioned above, the education backgrounds are likely to have an impact on the willingness to take risks. In my interpersonal circle, people generally have a high level of education, which may affect randomness.

Therefore, if we want to do further researches in this field in the future, we should try to improve the research method. If a questionnaire is again designed, for instance, more relevant questions should be added to make the results more convincing ( better to have proper rewards as an incentive, of course). In addition, efforts should be made to expand the source of respondents so as to ensure the randomness and objectiveness. To reach these, it is bound to face greater challenges and more preparatory work should be involved.

\section{CONCLUSION}

This article first introduces the proposal of Prospect Theory and Expected Utility Theory preceded and draws a hypothesis that leaders are more likely to take risks than non-leaders. And then I conduct a survey on this issue based partially on Prospect Theory and other findings about correlations between leadership effectiveness and motivations to lead. There are good responses and the result proves the correctness of my hypothesis. Next, I try to analyse the essence of this 
phenomenon by referring to some findings on different types of leaders and find that except those who are reluctant and less motivated to become leaders, more leaders are risk-loving than non-leaders both in gain and loss versions, provided that most of people are still generally risk-adverse in gain versions. The limitations of my study are also taken into account and analysed concretely. At last, a few possible approaches to improve the study are simply mentioned.

This article only provides an angle of characterizing how leaders and non-leaders probably make their decisions, and further studies aimed at this issue remain to be seen. Decision-making is persistent now and forever, and I believe that it is always beneficial to comprehend the intrinsic motivations of every decision made by different kinds of people so that better decisions can be made in any conditions.

\section{ACKNOWLEDGMENT}

Here I'm going to acknowledge all the respondents that have given support to my research by providing valid and authentic data in the questionnaires. And I'd like to thank Erica, my teaching assistance and Krishna, my teacher for their advice to improve my article. Besides, it's Prospect Theory that exploit a brand new way for me to go into something related to this, and its proposers, Daniel Kahneman and Amos Tversky, are who I would also be grateful to.

\section{REFERENCES}

[1] Dapeng Luan, Jian Wang. The Development and Latest Trends of Expected Utility Theory [J]. Tianfu New Idea, 2012(05): 36-41.

[2] Kahneman, Daniel, and Amos Tversky. "Prospect Theory: An Analysis of Decision under Risk." Econometrica, vol. 47, no. 2, 1979, pp. 263-291.

[3] Ziguo Ma. An introduction to several behavioral economics theories that has won Nobel Prizes [J]. Journal of Finance and Economics, 2018(16): 161-162.

[4] Xiaoling Zhong, Junbo Wang, Prospect theory and corporate bond returns: An empirical study, Journal of Empirical Finance, Volume 47, 2018, Pages 25-48, ISSN 0927-5398.

[5] Birgit Schyns, Tina Kiefer, Roseanne J. Foti, Does thinking of myself as leader make me want to lead? The role of congruence in self-theories and implicit leadership theories in motivation to lead, Journal of Vocational Behavior, Volume 122, 2020, 103477, ISSN 0001-8791.

[6] Badura, K. L., Grijalva, E., Galvin, B. M., Owens, B. P., \& Joseph, D. L. (2020). Motivation to lead: A meta-analysis and distal-proximal model of motivation and leadership. Journal of Applied Psychology, 105(4), 331-354.

[7] Xueting Jiang, Sen Xu, Jeffery D. Houghton, Steve J. Kulich, Leader self-development: Why do people develop themselves as leaders?, Business Horizons, 2020, ISSN 0007-6813.

[8] McDANIEL, M.A., HARTMAN, N.S., WHETZEL, D.L. and GRUBB, W.L., III (2007), SITUATIONAL JUDGMENT TESTS, RESPONSE INSTRUCTIONS, AND VALIDITY: A META-ANALYSIS. Personnel Psychology, 60: 63-91. 